\title{
Iron Overload and Iron-Chelation Therapy in Patients with Myelodysplastyc Syndromes
}

\author{
Lisette Del Corso, Eleonora Arboscello and Enrico Balleari* \\ Department of Hematology and Oncology; IRCCS AOU San Martino-IST, Genova, Italy
}

\begin{abstract}
Transfusion-dependency is an independent prognostic factor for survival in myelodysplastic syndromes (MDS). This negative impact is mainly due to the iron overload (IOL) consequent in large part to chronic transfusion therapy because of its detrimental effects on cardiac, hepatic and endocrine functions. The main tools useful for clinical diagnosis and monitoring of IOL should be serum ferritin, transferrin saturation and magnetic resonance imaging.

If correctly evaluated, IOL is frequently observed in lower-risk MDS patients, and is more pronounced in longer surviving individuals. A detrimental role of IOL in MDS patients has been documented in several studies, showing a correlation with a shorter overall survival. Treatment of IOL with iron chelating therapy (ICT) has been shown to prevent at least in part these negative effects. The goal of ICT in MDS patients is to prevent and treat complications of IOL and to improve survival.
\end{abstract}

Keywords: Iron overload; Myelodysplastic syndromes; Iron chelation therapy

\section{Introduction}

Myelodysplastic Syndromes (MDS) are a group of clonal disorders of hematopoietic stem cells, characterized by peripheral blood cytopenias with morphological dysplasia due to ineffective hematopoiesis, with a variable but consistent risk of progression to acute myelogenous leukemia (AML) [1]. MDS are one of the most common hematologic malignancies, with an incidence varying from 3 to 12 cases per 100,000 people per year in the general population, which increase up to 15-50 cases per 100,000 in older patients ( $>70$ years of age); the median age at diagnosis is around 75 years in most of the series [2].

Clinical course of MDS is extremely heterogeneous; many MDS patients have a life expectancy of several years, while others surviving only a few months. The International Prognostic Score System (IPSS), which is based on a combination of clinical, morphological and cytogenetic criteria, is at present the best known and most widespread system of prognostic classification of MDS [3]. According to the recently revised version of this scoring system (R-IPSS), 5 different groups of patients with an increasing risk of death or leukemic transformation are recognized [4]. Moving from the "very-low" up to the "very-high" risk, the median overall survival actually decreases from 8.8 years for the "very-low" risk-group down to 0.8 years for the "very-high" risk, respectively [4].

The clinical course of MDS is dominated by symptoms and signs of bone marrow failure. Anemia is the most common clinical feature of MDS: more than $50 \%$ of MDS patients at diagnosis present anemia and actually almost all of them develop anemia during the course of the disease [5].

Although chronic anemia is only seldom life-threatening, it can lead to significant morbidity and it has a negative impact on both performance status and overall survival [5]. In particular, anemia is per se an important factor of risk for cardiovascular events that often lead to both morbidity and death. In fact, more than $50 \%$ of MDS patients with a "lower risk" disease actually die from causes different from MDS itself, especially cardiac failure [5].

The majority of MDS patients during the course of the disease develop a symptomatic anemia requiring transfusions of packed red blood cells (PRBC), each of them containing approximately $200 \mathrm{mg}$ of elementary iron. Because of the fact that human beings are unable to actively eliminate iron from the body, iron overload develops almost inevitably in repetitively transfused patients, with a median assumption of about 4 to 8 grams of iron per year; secondary emosiderosis yet becomes evident when body iron content is above 7-14 grams, an amount easily reached after receiving as few as 15-30 PRCB units [6].

Iron overload is associated with a worse outcome of MDS patients, because of its negative impact on cardiac, hepatic and endocrine functions [6,7] and its prevention and/or correction deserves particular importance, especially in subjects with a longer life expectancy. Although transfusion-dependency is not the only cause of iron accumulation in aged and often frail MDS patients because iron accumulation often starts before they became transfusion-dependent [8], long-term transfusion therapy certainly is the most important cause of iron overload in these patients.

In this review we critically examine the clinical impact of iron overload on MDS patients and describe current available data concerning its treatment, with particular interest on iron chelating therapy (ICT).

\section{Evaluation of iron overload}

According to recent authoritative guidelines, the main tools useful for clinical diagnosis and monitoring of iron overload should be serum ferritin (SF), transferrin saturation and magnetic resonance (RM) imaging [9].

*Corresponding author: Enrico Balleari, Department of Hematology and Oncology; IRCCS AOU San Martino-IST, Largo R. Bensi 10, 16132 Genova, Italy, Tel: 00-39-010-5555780; Fax: 00-39-010-5556788; E-mail: balleari@unige.it

Received September 20, 2013; Accepted November 14, 2013; Published November 20, 2013

Citation: Corso LD, Arboscello E, Balleari (2013) Iron Overload and IronChelation Therapy in Patients with Myelodysplastyc Syndromes. J Blood Disorders Transf 5: 175. doi: 10.4172/2155-9864.1000175

Copyright: ( 2013 Lee Corso LD, et al. This is an open-access article distributed under the terms of the Creative Commons Attribution License, which permits unrestricted use, distribution, and reproduction in any medium, provided the original author and source are credited. 
Serum ferritin is a practical, inexpensive and readily accessible indicator of body iron burden, although additional methods are needed for a precise diagnosis of iron overload.

The primary toxic form of iron, the non-transferrin bound iron (NTBI), is detected when transferrin saturation exceeds $70 \%$ to $80 \%$. NTBI, generated when plasma iron exceeds the transferrin's binding capacity, combines with oxygen to form hydroxyl and oxygen radicals that are defined as "reactive oxygen species" (ROS), and these toxic elements cause lipid peroxidation, cell membrane protein DNA and ultimately organ damage [8]. A particular sub-fraction of NTBI with a strong capacity of catalyzing the generation of ROS is the so-called labile plasma iron (LPI) [10].

NTBI and possibly LPI may be a more specific indicator of iron overload than the SF [11], but their use in clinical practice is at present limited. On the other hand, although SF is an acute phase reaction protein and its levels may fluctuate in the short term in response to different factors such as infection and inflammation, long-term trends of SF affordably reflect underlying change in body iron stores and provide a useful and practicable tool for monitoring iron overload [12].

A precise quantification of iron overload in a single specific individual can be accurately obtained non-invasively by assessing hepatic and cardiac iron content using T2* MR Imaging (T2* MRI) [13]. $\mathrm{T} 2^{\star}$ represents the echo time necessary for a tissue to become twice as dark. It may be thought of as a half-life, with small values representing rapid signal loss. Alternatively, image darkening can be expressed by $\mathrm{R} 2^{*}$, its rate of darkening. Some investigators prefer to report R2* values rather than $\mathrm{T} 2^{*}$ values, because $\mathrm{R} 2^{*}$ is directly proportional to iron concentration [14]. T2* MRI has already been used to quantify heart, liver and endocrine iron in transfusion-dependent MDS patients [15]. Although there is no linear correlation between hepatic and cardiac iron overload [14], and iron toxicity may not only depend on the degree of tissue iron accumulation but it might also be related to the extent of chronic exposure to NTBI [16], cardiac assessment by T2* MRI allows in vivo estimation of iron content, and, more important, is specific for iron overload and unaffected by confounding factors.

\section{Iron overload and Mds}

If correctly evaluated, iron overload is frequently observed in lower-risk MDS patients, especially in those patients with a long-term history of anemia requiring chronic support of PRCB transfusions. From a pathophysiologic point of view, long-term transfusion therapy is certainly the most important cause of iron overload in these patients; nevertheless, others mechanism(s) account for this phenomenon, and in particular the role of the ineffective erythropoiesis. In MDS patients, in fact, ineffective erythropoiesis which is seen in pure erythroid disorders such as refractory anemia (RA) with or without ringed sideroblasts, determines suppression of hepcidin production by hepatocytes [8] Low hepcidin levels increase iron absorption from the intestine and increase iron release from macrophages, which can occur even when iron levels are already elevated [17].

The exact clinical impact of iron overload on morbidity and mortality in regularly transfused MDS patients is not clearly demonstrated.

The effects of iron overload in MDS transfusion-dependent patients have been analyzed in a retrospective analysis of 467 MDS patients [8]. In this study, iron overload had a relevant negative impact on clinical outcome of this cohort of patients, in whom cardiac disease related mortality and hepatic cirrhosis were the two more frequent cause of death (51\% and $8 \%$ respectively). Actually, MDS patients who died from cardiac or hepatic failure had received a significantly higher number of PRBC units than those who died from other causes. Iron overload seems therefore to closely correlate to cardiac failure and mortality, although it is difficult to determine how much cardiac risk is attributable to iron overload, because MDS patients are usually older and have several comorbidities that may precipitate cardiac risk [18]. However, experience in thalassemia major patients clearly demonstrates that cardiac $\mathrm{T} 2{ }^{*}$ is associated with systolic and diastolic ventricular dysfunction [19]. A recent cross-sectional study in 75 consecutive regularly transfused MDS patients assessing cardiac iron overload by $\mathrm{T} 2^{*} \mathrm{RMI}$ demonstrated a cardiac iron overload $\left(\mathrm{T} 2^{*}>20 \mathrm{~ms}\right)$ in $18.2 \%$ of patients, and an inverse correlation between the number of PRBC units transfused and cardiac T2* value was found [20]. In the same study, the incidence of severe cardiac dysfunction as evaluated by echocardiography was significantly higher MDS patients with $\mathrm{T} 2{ }^{*}$ $>20 \mathrm{~ms}$, thus indicating that iron overload impairs systolic function in regularly transfused MDS patients [20].

Iron overload could impair survival in MDS not only through organ damage due to iron accumulation, but also by accelerating progression to AML [21]. An increase of toxic reactive oxygen compounds generated by free iron has been shown to increase oxidative cellular stress and thus probably it might contribute to ineffective hematopoiesis and genetic instability of the MDS clone [22]. The risk of AML progression of MDS patients was correlated with iron overload and transfusion-dependency in a multivariate analysis evaluating the clinical factors possibly related with leukemic evolution [23], and ICT has been shown to synergize in vitro with vitamin $\mathrm{D}$ to promote monocyte differentiation in primary AML cells. [24]

Finally, the increase in free iron that results from iron overload conditions is a well-established risk factor for invasive fungal infection (IFI) such as mucormycosis or aspergillosis, which account for a significant proportion of deaths, in particular in "higher-risk" MDS patients undergoing intensive chemotherapy or hematopoietic stem cell transplantation [25].

\section{Management of Iron Overload in Mds}

Complications of transfusional iron overload may be prevented and treated by an efficient ICT. Iron chelators are agents that promote negative iron balance by binding and allowing excretion of iron in a non-toxic form. Three iron chelators who can prevent or reduce iron overload are at present available for clinical use: deferoxamine (DFO), deferiprone (DFP) and deferasirox (DFX), and are utilised for ICT in clinical practice. DFO was the first chelator introduced in the clinical use for many years [26]. DFO retains indeed not a good compliance because it is not orally available but requires long-term intravenous or subcutaneous administration. DFP and DFX are oral chelators given three and one time a day, respectively. DFP is usually avoided in MDS patients and in individuals with aplastic anemia, due to its potential to induce agranulocytosis [27]. Due to the proven efficacy, oral administration and favorable pharmacokinetics, DFX is presently recognized as the first-choice agent for ICT in several guidelines [12,28]. The starting daily dose of FDX is $10-20 \mathrm{mg} / \mathrm{Kg}$ and its dosage should be adjusted according to the transfusional regimen, SF concentrations and organ damage.

In general, the goal of ICT is to remove excess of iron from the body in order to prevent or reduce its accumulation in the tissue. The benefits of ICT for many years have been clearly demonstrated in transfusiondependent patients with thalassemia [29], and are more and more convincingly reported also in MDS [30,31]. 
The goal of ICT in MDS patients with iron overload is to prevent and treat complications of iron overload and to improve survival. At present, the role of ICT in MDS is largely speculative, due to the absence of sufficiently large, prospective and randomized studies. However, several small retrospective studies reported a beneficial effect of regular ICT on survival and on the rate of leukemic transformation in MDS patients [32,33].

Two more recent and larger clinical studies evaluated the efficacy of DFX on 341 and 173 MDS patients, respectively [34,35]. In both these studies data demonstrated that DFX effectively reduced serum ferritin, hepatic damage and may contribute to increase cytopenias.

While there is therefore an increasing and quite clear evidence of ICT efficacy on the reduction of iron overload in transfusion-dependent MDS subjects, the effects of this treatment on overall survival of transfusion-dependent MDS patients remain to be demonstrated. A retrospective study conducted by Rose et al. on 97 patients with lowrisk or intermediate-1 MDS treated with ICT (mostly DFO) indeed demonstrated a better overall survival for ICT treated patients than untreated. The positive impact of ITC on survival of MDS patients may not only depend on the prevention of organ damage, but also to the decrement on infections and leukemogenesis [30].

Some emerging evidences indicate that ICT can actually improve hematopoiesis and lead to a reduction or abolition of transfusiondependence in MDS patients. Positive effects on hematopoiesis were anecdotally described in the past in subjects with MDS treated with DFO or DFP. Actually, in 1996 Jensen et al. for the first time demonstrated that ICT can improve the transfusion need in MDS patients [36]. In more recent years, after the introduction of DFX in the clinical practice, several small series or single case reports confirmed that ICT decreases transfusion request in MDS patients [37-40]. Accordingly to these reports, the EPIC trial, a prospective 1 year multicenter open-label study on the effects of DFX treatment in 1744 patients with transfusiondependent anemias, showed that in the subset of MDS patients erythroid responses -as defined according to IWG 2000 criteria [41] were observed in $21.5 \%$ of patients, with a median time to response of 109 days [42].

The mechanism(s) by which ICT may lead to hematologic improvement are unclear. The reduction of iron store induced by ICT seems to up regulate erythropoietin response, resulting in hemoglobin increase [43], and a recent in vitro study showed that DFX is a potent inhibitor of nuclear factor kappa-light-chain-enhancer of activated B cells, which is abnormally activated in MDS blast cells [44]. Other off target effects of DFX by which it can improve erythropoiesis are an increase of chemosensitivity, a decrease of fungal infection rate, mTOR inhibition [45], and a reduction of oxidative species, which are believed to correlate with inefficient erythropoiesis [46].

Interestingly, a recent retrospective case-control study showed that DFX synergizes with vitamin D to promote monocyte differentiation, and it may increase the overall survival in elderly patients (10.4 months versus 4 months) [24]. Several recent practice guidelines $[12,28]$ therefore state ITC as recommended treatment in low-risk and transfusion-dependent MDS patients with iron overload and in patients with high-risk disease when they are responders to therapies able to modify their life expectancy or have a HSCT in their program. The decision to initiate ICT is usually taken after considering the number of transfusions received as well as the measured iron burden. The rate rather than the absolute number of PRBC units received may be more relevant to the decision to start ICT in MDS patients. In fact, an important consideration in deciding which patients are likely to benefit most from ICT is the degree of transfusion-dependency. MDS patients with significant transfusion dependency are most likely to need ICT; in terms of iron burden, elevated SF levels or other evidence (T2* RMI) of tissue iron overload are probably good indicators of a benefit of ICT. Inception of ICT should not be decided uniquely on the basis of serum ferritin level $(<1000 \mu \mathrm{g} / \mathrm{ml})$ but it may be considered also after they received just 10 to 20 red cell units. Another key consideration includes the patient's life expectancy. Even if in patients with a short life expectancy ICT may not be justified because iron overload-related complications may not have the time to emerge, it should be considered that ICT may in many cases reverse existing complications due to iron overload.

\section{Conclusions}

Iron overload is a problem of relevant importance in MDS patients, especially for them with a lower-risk disease or candidate for a bone marrow transplant.

ICT may improve quality of life and perhaps survival in MDS patients and should be aimed at preventing cardiac and hepatic damage due to transfusional overload in all patients with lower-risk MDS with a life expectancy $>1$ year, and who have received 20 red blood cell units.

New prospective clinical studies addressing this topic are at present ongoing and will be able to define in the near future the exact role of ICT on the management of iron overload in patients with MDS, but still now this treatment has to be considered as an important therapeutic option for an optimal management of transfusion-dependent MDS patients with iron overload.

\section{References}

1. Aul C, Bowen DT, Yoshida Y (1998) Pathogenesis, etiology and epidemiology of myelodysplastic syndromes. Haematologica 83: 71-86.

2. Ma X (2012) Epidemiology of myelodysplastic syndromes. Am J Med 125: S2-5.

3. Greenberg P, Cox C, LeBeau MM, Fenaux P, Morel P, et al. (1997) International scoring system for evaluating prognosis in myelodysplastic syndromes. Blood 89: $2079-2088$

4. Greenberg PL, Tuechler H, Schanz J, Sanz G, Garcia-Manero G, et al (2012) Revised international prognostic scoring system for myelodysplastic syndromes. Blood 120: 2454-2465.

5. Malcovati L, Della Porta MG, Strupp C, Ambaglio I, Kuendgen A, et al. (2011) Impact of the degree of anemia on the outcome of patients with myelodysplastic syndrome and its integration into the WHO classification-based Prognostic Scoring System (WPSS). Haematologica 96: 1433-1440.

6. Malcovati L (2007) Impact of transfusion dependency and secondary iron overload on the survival of patients with myelodysplastic syndromes. Leuk Res 31 Suppl 3: S2-6.

7. Oliva EN, Dimitrov BD, Benedetto F, D'Angelo A, Nobile F (2005) Hemoglobin level threshold for cardiac remodeling and quality of life in myelodysplastic syndrome. Leuk Res 29: 1217-1219.

8. Gattermann N, Rachmilewitz EA (2011) Iron overload in MDS-pathophysiology, diagnosis, and complications. Ann Hematol 90: 1-10.

9. Remacha A, Sanz C, Contreras E, De Heredia CD, Grifols JR, et al. (2013) Guidelines on haemovigilance of post-transfusional iron overload. Blood Transfus 11: 128-139.

10. Cabantchik ZI, Breuer W, Zanninelli G, Cianciulli P (2005) LPI-labile plasma iron in iron overload. Best Pract Res Clin Haematol 18: 277-287.

11. Brissot P, Ropert M, Le Lan C, Loréal O (2012) Non-transferrin bound iron: a key role in iron overload and iron toxicity. Biochim Biophys Acta 1820: 403-410.

12. Greenberg PL, Attar E, Bennett JM, Bloomfield CD, De Castro CM, et al. (2011) National Comprehensive Cancer Network. NCCN Clinical Practice Guidelines in Oncology: myelodysplastic syndromes. J Natl Compr Canc Netw 9:30-56. 
Citation: Corso LD, Arboscello E, Balleari (2013) Iron Overload and Iron-Chelation Therapy in Patients with Myelodysplastyc Syndromes. J Blood Disorders Transf 5: 175. doi: 10.4172/2155-9864.1000175

13. Carpenter JP, He T, Kirk P, Roughton M, Anderson LJ, et al (2011) On T2* magnetic resonance and cardiac iron. Circulation 123: 1519-1528.

14. Wood JC (2011) Impact of iron assessment by MRI. Hematology Am Soc Hematol Educ Program 2011: 443-450.

15. Chacko J, Pennell DJ, Tanner MA, Hamblin TJ, Wonke B, et al. (2007) Myocardial iron loading by magnetic resonance imaging $\mathrm{T} 2 *$ in good prognostic myelodysplastic syndrome patients on long-term blood transfusions. $\mathrm{Br} J$ Haematol 138: 587-593.

16. Hider RC (2002) Nature of nontransferrin-bound iron. Eur J Clin Invest 32 Suppl 1: $50-54$.

17. Nemeth E (2008) Iron regulation and erythropoiesis. Curr Opin Hematol 15 169-175.

18. Della Porta MG, Malcovati L (2009) Clinical relevance of extra-hematologic comorbidity in the management of patients with myelodysplastic syndrome. Haematologica 94: 602-606.

19. Pennell DJ (2005) T2* magnetic resonance and myocardial iron in thalassemia Ann N Y Acad Sci 1054: 373-378.

20. Pascal L, Beyne-Rauzy O, Brechignac S, Marechaux S, Vassilieff D, et al. (2013) Cardiac iron overload assessed by $\mathrm{T}^{*}$ magnetic resonance imaging and cardiac function in regularly transfused myelodysplastic syndrome patients. Br J Haematol 162: 413-415.

21. Chan LSA, Buckstein R, Reis MD, Chesney A, Lam A, et al. (2008) Iron overload and haematopoiesis in MDS: does blood transfusion promote progression to AML? Blood 112

22. Rassool FV, Gaymes TJ, Omidvar N, Brady N, Beurlet S, et al. (2007) Reactive oxygen species, DNA damage, and error-prone repair: a model for genomic instability with progression in myeloid leukemia? Cancer Res 67: 8762-8771.

23. Sanz G, Nomdedue B, Teresa B, Mohamed B, Such E, et al. Independent impact of iron overload and transfusion dependency on survival and leukemic evolution in patients with myelodysplastic syndrome. Blood 112

24. Paubelle E, Zylbersztejn F, Alkhaeir S, Suarez F, Callens C, et al. (2013) Deferasirox and vitamin $D$ improves overall survival in elderly patients with acute myeloid leukemia after demethylating agents failure. PLoS One 8: e65998.

25. Majhail NS, Lazarus HM, Burns LJ (2008) Iron overload in hematopoietic cel transplantation. Bone Marrow Transplant 41: 997-1003.

26. Propper RD, Shurin SB, Nathan DG (1976) Reassessment of the use of desferrioxamine B in iron overload. N Engl J Med 294: 1421-1423.

27. Cohen AR, Galanello R, Piaga A, Dipalma A, Vullo C, et al. (2000) Safety profile of the oral iron chelator deferiprone: a multicenter study. $\mathrm{Br} \mathrm{J}$ Haematol 108 305-312.

28. Santini V, Alessandrino PE, Angelucci E, Barosi G, Billio A, et al. (2010) Clinical management of myelodysplastic syndromes: update of SIE, SIES, GITMO practice guidelines. Leuk Res 34: 1576-1588.

29. Capellini MD (2008) Long-term efficacy and safety of deferasirox. Blood Reviews 22: 535-541.

30. Rose C, Brechignac S, Vassilief D, Pascal L, Stamatoullas A, et al. (2010) Does iron chelation therapy improve survival in regularly transfused lower risk MDS patients? A multicenter study by the GFM (Groupe Francophone des Myélodysplasies). Leuk Res 34: 864-870.

31. Gattermann N, Finelli C, Porta MD, Fenaux P, Ganser A, et al. (2010) Deferasirox in iron-overloaded patients with transfusion-dependent myelodysplastic syndromes: Results from the large 1-year EPIC study. Leuk Res 34: 1143-1150.

32. List AF, Baer MR, Steensma D, Raza A, Esposito J, et al. (2009) Twoyear analysis of efficacy and safety of deferasirox (Exjade) treatment in myelodysplastic syndrome patients enrolled in the US03 Study. Blood 114 3829.
33. Molteni A, Riva M, Speziale V, Morra E, Cappellini MD (2010) Iron-chelation therapy in MDS/IMF patients: does it really impact on transfusion requirement? Haematologica 95:566.

34. Cappellini MD, Porter J, El-Beshlawy A, Li CK, Seymour JF, et al. (2010) Tailoring iron chelation by iron intake and serum ferritin: the prospective EPIC study of deferasirox in 1744 patients with transfusion-dependent anemias. Haematologica 95: 557-566.

35. List AF, Baer MR, Steensma DP, Raza A, Esposito J, et al. (2012) Deferasirox reduces serum ferritin and labile plasma iron in $\mathrm{RBC}$ transfusion-dependent patients with myelodysplastic syndrome. J Clin Oncol 30: 2134-2139.

36. Jensen PD, Heickendorff L, Pedersen B, Bendix-Hansen K, Jensen FT, et al. (1996) The effect of iron chelation on haemopoiesis in MDS patients with transfusional iron overload. $\mathrm{Br} J$ Haematol 94: 288-299.

37. Messa E, Cilloni D, Messa F, Arruga F, Roetto A, et al. (2008) Deferasirox treatment improved the hemoglobin level and decreased transfusion requirements in four patients with the myelodysplastic syndrome and primary myelofibrosis. Acta Haematol 120: 70-74.

38. Breccia M, Loglisci G, Salaroli A, Cannella L, Santopietro M, et al. (2010) Deferasirox treatment interruption in a transfusion-requiring myelodysplastic patient led to loss of erythroid response. Acta Haematol 124: 46-48.

39. Guariglia R, Martorelli MC, Villani O, Pietrantuono G, Mansueto G, et al. (2011) Positive effects on hematopoiesis in patients with myelodysplastic syndrome receiving deferasirox as oral iron chelation therapy: a brief review. Leuk Res 35: 566-570.

40. Molteni A, Riva M, Pellizzari A, Borin L, Freyre A, et al. (2013) Hematological improvement during iron-chelation therapy in myelodysplastic syndromes: the experience of the "Rete Ematologica Lombarda". Leuk Res 37: 1233-1240.

41. Cheson BD, Bennett JM, Kantarjian H, Pinto A, Schiffer CA, et al. (2000) Report of an international working group to standardize response criteria for myelodysplastic syndromes. Blood 96: 3671-3674.

42. Gattermann N, Finelli C, Della Porta M, Fenaux P, Stadler M, et al. (2012) Hematologic responses to deferasirox therapy in transfusion-dependent patients with myelodysplastic syndromes. Haematologica 97: 1364-1371.

43. Vreugdenhil G, Smeets M, Feelders RA, van Eijk HG (1993) Iron chelators may enhance erythropoiesis by increasing iron delivery to haematopoietic tissue and erythropoietin response in iron-loading anaemia. Acta Haematol 89: 57-60.

44. Messa E, Carturan S, Maffè C, Pautasso M, Bracco E, et al. (2010) Deferasirox is a powerful NF-kappaB inhibitor in myelodysplastic cells and in leukemia cell lines acting independently from cell iron deprivation by chelation and reactive oxygen species scavenging. Haematologica 95: 1308-1316.

45. Ohyashiki JH, Kobayashi C, Hamamura R, Okabe S, Tauchi T, et al. (2009) The oral iron chelator deferasirox represses signaling through the mTOR in myeloid leukemia cells by enhancing expression of REDD1. Cancer Sci 100: 970-977.

46. Ghoti H, Amer J, Winder A, Rachmilewitz E, Fibach E (2007) Oxidative stress in red blood cells, platelets and polymorphonuclear leukocytes from patients with myelodysplastic syndrome. Eur J Haematol 79: 463-467. 